\title{
Rational Agent-Based Decision Algorithm for Strategic Converged Network Migration Planning
}

\author{
Sai Kireet Patri, (D) Elena Grigoreva, Wolfgang Kellerer, (i) and Carmen Mas Machuca
}

\begin{abstract}
To keep up with constantly growing user demands for services with higher quality and bandwidth requirements, telecommunication operators are forced to upgrade their networks. This upgrade, or migration of the network to a new technology, is a complex strategic network planning problem that involves technoeconomic evaluations over multiple periods of time. The state-of-the-art approaches consider migrations to a concrete architecture and do not take uncertainties, such as user churn, into account. This results in migration cost underestimations and profitability overestimations. In this paper, we propose a generic migration algorithm derived from a search-based rational agent decision process that can deal with uncertainties and provides the migration path using a maximized utility function. The algorithm maximizes the migration project profitability, measured as the accumulated net present value. This flexible and generic methodology has been evaluated on the example of migration from existing copper networks to the future-proof passive optical network architectures. Our proposed flexible migration algorithm is validated over pure residential and converged scenarios in a fully reproducible case study. The results affirm that migration flexibility is key to profit maximization.
\end{abstract}

Index Terms-Artificial intelligence (AI); Converged network planning; Migrations; Net present value (NPV); Network planning; Passive optical network (PON); Rational agents; Strategic network planning.

\section{INTRODUCTION}

B andwidth-hungry services are becoming a daily reality for every household. Online gaming, HD-TV, and an overall growth of employees working remotely from their homes challenge the network operator with ever growing bandwidth requirements. The operators are forced to upgrade the network by the competition and governmental initiatives [1], while fighting to maintain a sufficient gap between costs and the revenues. This upgrade, or migration, to the next technology, while satisfying user and

Manuscript received February 11, 2019; revised May 2, 2019; accepted May 23, 2019; published June 26, 2019 (Doc. ID 359961).

S. K. Patri (e-mail: kireet.patri@tum.de) is with ADVA Optical Networking SE and a member of the research team at the Chair of Communications Networks, Technical University of Munich, Munich, Germany.

E. Grigoreva, W. Kellerer, and C. Mas Machuca are with the Chair of Communications Networks, Technical University of Munich, Munich, Germany.

https://doi.org/10.1364/JOCN.11.000371 regulatory requirements, calls for careful strategic network planning to fulfill the requirements, while maximizing the benefits of the operator [2]. In general, strategic network planning is done as a telecommunication project evaluation, which makes it possible to choose suitable technology, evaluate the risks, and plan the network upgrades, i.e., migrations.

A migration project (from here on referred to simply as a "project") consists of a multidimensional, multiperiod planning problem, and its solution involves market penetration forecasting, dimensioning of the network infrastructure and processes, and evaluation of their total cost of ownership (TCO) [3]. Due to migration planning complexity and lack of open network TCO models, the state-of-the-art migration models are case-study specific, focusing either on only a single-migration technology or a single-migration path. Throughout this work, the term migration path refers to the technology sequence (in time) from the starting technology to the goal of the migration.

Over the years, tree-based artificial intelligence (AI) algorithms have emerged as a frontrunner to solve problems in which complete information is not available. In the case of access network migration, this maps to providers making strategic decisions as to when and how many migration steps are needed to maximize the profits, keeping in mind an uncertain number of subscribers, variations on revenues, and also the associated TCO. The solution proposed in this work is based on the Expectimax search algorithm [4], which is commonly used in AI-based applications, and for the first time, to the best of our knowledge, it has been applied to the network migration problem.

These algorithms usually consist of (1) agents, which execute an action based on the environment, and (2) an environment which changes based on agent actions. Any agent who takes an action to maximize its performance metric, is said to be a rational agent [5].

The contribution of this paper is as follows. First, we propose a generic rational agent-based algorithm to maximize the migration project profitability while taking into account user uncertainty. The introduced algorithm makes migration decisions (when to migrate to which technology) keeping in mind the migration goal, which in our case is to maximize the project profitability over the network lifetime. This algorithm aims to serve as a strategic analysis tool to help network planners, researchers, and industry 
managers to make decisions, compare solutions, estimate time and costs, and evaluate the impact of new solutions. The proposed algorithm is validated with realistic case studies: migrating from copper-based networks to passive optical networks (PONs) in different deployment areas (rural, urban, and dense urban) and user mixes (residential and converged planning). The validation includes creating a database of PON technologies and architectures [6], calculating their realistic costs based on geographical data (using the network planning tool available [7]), and finally, performing a comprehensive sensitivity analysis to validate the assumptions.

Our case-study results show that the network operators can achieve higher profitability while providing the required data rates to different subscribers with the flexible migration end state. In this case, the final state is chosen by the migration algorithm to maximize economic value instead of targeting a concrete technology. Furthermore, accounting for user churn avoids overestimating the project profitability. In this paper, we present selected results for the urban area; the full set of results can be found in [6]. Last but not least, an important contribution of this paper is the full reproducibility of the study, because all the implementations are publicly available $[7,8]$, including a complete database of the technoeconomic parameters [6].

The rest of this paper is organized as follows. Section II deals with the current state of the art for the various approaches to migration planning in access networks. Section III describes the problem formulation and assumptions for the migration algorithm. It lists and explains all the necessary inputs to conduct a migration study. Section IV introduces the migration methodology based on an uninformed-search algorithm. Section V shows the results [expected net present values (NPVs) and migration paths] of selected scenarios and the sensitivity analysis. Finally, Section VI presents the conclusions and outlook.

\section{State of the ART}

Optical access network (OAN) technologies provide, among other benefits, longer reach and higher sustainable data rates, which make them an ideal candidate to provide high-speed data rates to customers [9]. However, these OANs come in various configurations, each with its own type of equipment and dimensioning. A simple modeling of costs cannot suffice in finding the best technology to be deployed. Because deployment of OANs lasts over a long period of time (between 5 and 10 years), an analysis involving the time value of money is required to find a cost-effective solution.

The authors of [9] undertake a cost-benefit analysis of various OAN technologies, assumed to be deployed in a fiber-to-the-home (FTTH) configuration, as shown in Fig. 1. However, the possible benefits of fiber-to-the-building (FTTB) and fiber-to-the-cabinet (FTTCab) deployments are not covered. Also, the lack of granularity in operational expenditures (OPEX) calculations and the use of geometric models instead of geographical models for the network deployment lead to unpredictable TCO under- and

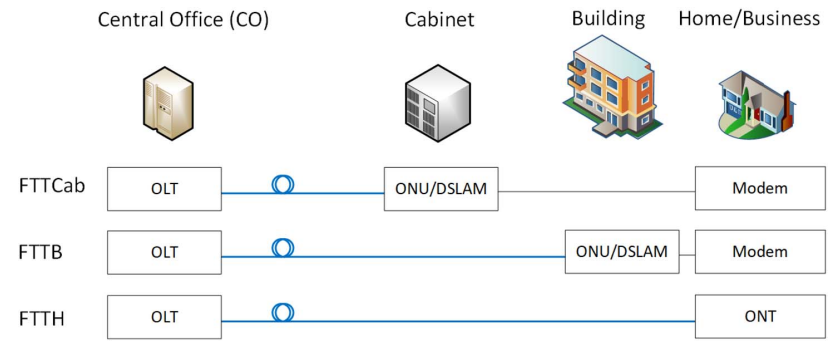

Fig. 1. Fiber-to-the-x (FTTx) schematics where $\mathrm{x}$ stands for the optical fiber termination point, which could be a cabinet (FTTCab), building (FTTB), or home (FTTH).

overestimations [10]. Another work applied to FTTH (i.e., without the possibility to choose the final migration state) is proposed in [3]. The authors contribute to planning of multistep migration when multiple technologies are present. The output is then an optimal migration path, which considers a migration window and holding time in every intermediate technology. However, the solution does not deal with the uncertainties such as user churn.

The use of AI based heuristic search in AON migration planning is studied in the work done in $[11,12]$. The problem statement defined in that work is to optimize the migration process from VDSL to FTTC GPON in an urban access network, undertaken in a predefined migration period. However, no evidence is provided if this optimization suffices for multistep migration when different migration options are present.

Another approach to identify the best network migration was undertaken in [13] using strategic analysis of a real options approach [14]. In the studied business case of a fiber-to-the-x (FTTx) migration from a full copper deployment, this translates to options like the size of cabinets to deploy and the services to be provided to subscribers, where all the options form a decision tree. However, the authors focus on a specific case study and model the probability based on assumptions made about the per-cabinet take-up rate, which cannot be generalized.

From the literature, the need for a migration model that provides the flexibility of choosing different access network deployment options in various scenarios while catering for revenue uncertainty is evident. To tackle this, we use the Expectimax search [4], which is a simplified uninformed adversarial search tree used to model sequential games. This search method is different from informed or deterministic searches, like MinMax search [4], because it considers the uncertainty arising from input parameters for which complete information is not available. In our work, we prefer an uninformed search over a deterministic search because it allows us to model subscriber uncertainty in the future.

Expectimax search allows us to have a stochastic model for this uncertainty by maximizing the probabilistic average over all possible outcomes over time to find an optimal solution. Its tree-based structure allows for three different nodes, namely, maximizer nodes (which find the maximum of its children), chance nodes (which find the probabilistic 
average of its children), and terminal nodes (which signify the end of tree building). An interested reader may refer to Section 2.3.3 in [6] and lecture notes in [4] for further indepth discussion.

\section{Problem Formulation, Assumptions, and InPut}

This section introduces the problem formulation and lists and explains the assumptions and necessary input for the model. Furthermore, this section introduces the economic parameters used to evaluate the results.

\section{A. Problem Formulation}

For better understanding, we explain the migration problem on a realistic example: migration from a copperbased asymmetric digital subscriber line 2+ (ADSL2+) network to PON. As has already been established in Section I, migration of access networks is a multidimensional and complex process and gives the network operator multiple options to explore.

With no loss of generality, to limit the scope of the paper we concentrate on the business model of the vertically integrated operator (VIO) [15]. We define the VIO to be the only Internet provider in a given area, who owns the physical infrastructure (fiber, ducts, remote nodes), network (OLTs, ONUs, splitters), and the services (Internet, television, telephony). The VIO assumes copper-based technologies are already deployed in an area and looks to migrate to any PON-based technology, shown in Fig. 2, because these are now mature and affordable to deploy $[16,17]$. The complete migration is limited in time, which is referred to as the migration window $T_{\text {mig }}$ [3]. However, the cost and revenue calculations take the network life cycle into account (usually longer than $T_{\mathrm{mig}}$ ), which is denoted by $T_{\mathrm{NW}}$.

The business model also involves the inclusion of different types of subscriber demands, such as a residential, business, and public intelligent transportation system (ITS) LTE macrobase stations (MBSs). With these, we create two different planning scenarios, namely, pure residential (i.e., connecting residential users) and converged (i.e., connecting residential, business, and ITS MBS demands). In the converged planning scenario, we ensure that the ITS

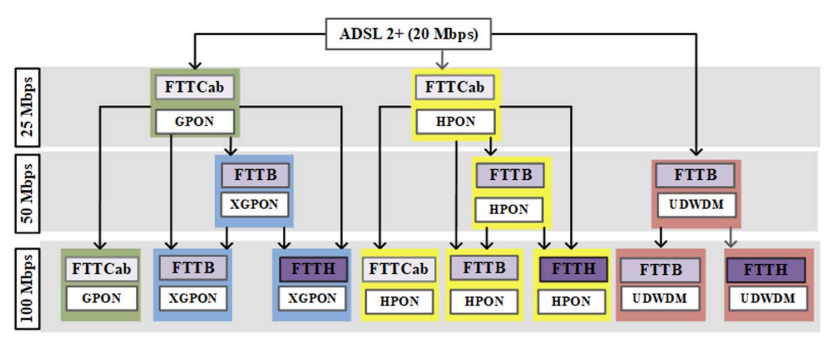

Fig. 2. Schematics of the allowed migrations starting from an ADSL 2+ solution offering $20 \mathrm{Mbps}$ to different solutions offering $100 \mathrm{Mbps}$, differing on the FTTx architecture, data rates, and technology.
MBSs are assigned separate wavelengths to support their backhaul traffic for high bandwidth and security purposes.

For different PON technologies, deployments can vary based on how far the fiber drop point is from the central office (CO). As shown in Fig. 1, FTTCab has an optical network unit (ONU) placed at street cabinets, whereas FTTB has a fiber drop point directly inside the building. This invokes a difference in civil works cost, which as described in further sections, is an important cost driver. Another important cost driver is the cost of active equipment at the $\mathrm{CO}$, which using the same PON deployment can achieve higher data rates.

Depending on the type of technology used, a gigabit Ethernet passive optical network (GPON), a 10 gigabit Ethernet passive optical network (XGPON), an ultradense wavelength division multiplexing passive optical network (UDWDM-PON), or a hybrid passive optical network (HPON) can be deployed [16]. Each of these technologies has different network equipment costs.

PON architectures can offer subscribers data rates of 25 , 50, and $100 \mathrm{Mbps}$ as shown in Fig. 2. The data rate can be increased incrementally by adding more active equipment at the $\mathrm{CO}$, making use of the dark fiber, and by blowing additional fiber through a duct, which was assumed to be less expensive as compared with digging and closing the ducts again [18].

For consistency, each scenario is referred to as a FTTx technology_bitrate. For example, FTTCab_GPON_25 refers to the FTTCab solution using GPON and offering $25 \mathrm{Mbps}$ to the end users. This way, using the migration tree shown in Fig. 2, we are able to find 13 different PON deployments, for example, FTTB_XGPON_50 and FTTB_UDWDM_100.

Figure 2 also defines allowed migration paths to achieve $100 \mathrm{Mbps}$ deployment for residential users, business users, and ITS MBSs. Some migrations, for example, migrating from FTTCab_GPON_25 to FTTH_UDWDM_100 or FTTB_UDWDM_50 to FTTB_XGPON_100 are restricted. This arises from our assumption that GPON- and XGPONbased technologies need a two-stage deployment (at least two remote nodes) to provide $100 \mathrm{Mbps}$ to users, whereas UDWDM technologies can satisfy the same requirement using a single-stage deployment $[6,16]$. Migrations involving changes in deployment types would lead to a higher TCO and can be avoided by restricting migrations between similar deployment types.

In this paper, we evaluate two scenarios, both of which have to provide at least $100 \mathrm{Mbps}$ per user [19]:

\section{1) Comparison scenario: fixed migration goal: FTTH}

Because most of the state-of-the-art papers consider a fixed final state as FTTH architectures, e.g., [3,9], this scenario considers only FTTH-based PON technologies providing the end user with $100 \mathrm{Mbps}$.

2) Proposed scenario: flexible migration goal: any FTTx that maximizes the profitability

All FTTx architectures, i.e., FTTCab, FTTB, and FTTH, that provide the subscribers with $100 \mathrm{Mbps}$ connection are considered. 
Comparing the results of these two scenarios, we evaluate the influence of the final state, i.e., fixed (only FTTH) or flexible (any FTTx) final state, when it is chosen only based on the maximum NPV at the end of the network life cycle. Because NPV requires a cost modeling of each of the different kinds of deployments, we need to find the TCO and define a revenue model, which is an input to the migration algorithm.

\section{B. Total Cost of Ownership and Revenue Model}

To make any business decisions, the cost of each option has to be evaluated. The TCO of the network consists of capital and operational expenditures (CAPEX and OPEX, respectively). This paper is neither focused on the TCO calculation nor the cost modeling, but on the migration modeling and evaluation. However, because the TCO is crucial to selecting the best migration scenario, we introduce the models, values, and methodology. We closely follow the approach in [9] and supplement it with values from $[16,20]$.

The CAPEX is split into two cost categories: civil works and equipment cost. The civil work cost is driven by the duct lengths and cost per meter of trenching and laying ducts. To find the costs of the network components, the respective electronic [optical line terminal (OLT) card, power splitter, digital subscriber line access multiplexer (DSLAM), ONU] and nonelectronic [fiber lengths, cabinets, heating ventilation and air conditioning (HVAC)] components are dimensioned based on the demands of the subscribers and the capacity of each component. The OPEX includes energy, fault management (FM), network operation, marketing, and rent. A detailed CAPEX and OPEX calculation used in this paper is presented in [6]. It is important to note that throughout this work, we present all costs and revenues in cost unit (C.U.), where 1 C.U. is fixed at the price of a single GPON ONU in the year 2013 [16].

The lengths of fibers and ducts for each of the deployments are obtained using the automated geography-based fixed network planning tool [7,21], which is based on the ESRI ArcMap 10.3.1 [22]. The tool calculates the duct and fiber lengths of feeder, distribution, and last mile fibers for the chosen area and network architecture. The planning is done on the real geographical street-based topologies to guarantee meaningful results for the TCO calculations. The planning details are out of the scope of this paper but are presented in detail in [6].

We model the subscriber behavior with three different penetration curves, as proposed in [17]: "conservative," "realistic," and "aggressive," which differ on the subscriber joining rates. These joining rates are then used to derive the number of subscribers connected to the network in every year, which is helpful in finding the yearly revenue generated in an area. Figure 3 shows the percentage of households connected in a pure residential scenario in a given year. We limit the technoeconomic calculations to 20 years because projects in a given area last between

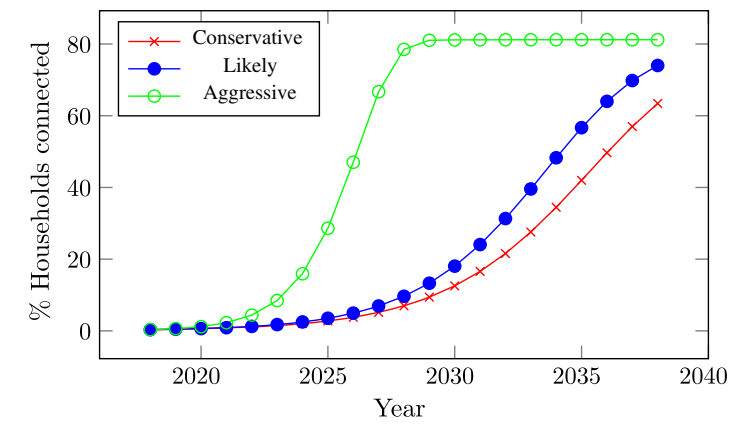

Fig. 3. Percentage of yearly connected residential subscribers based on the penetration curves from [17].

5 and 10 years, and the remaining years are used to recover costs.

The average revenue per user (ARPU), shown in Table I, reflects the real optical access market tariffs [1]. For business and ITS subscribers, the yearly generated revenues are assumed to be higher because these subscribers need separate hardware and wavelengths for better security and faster fault reparation [23]. Because the subscriber pays for the data rate, the ARPU does not change across different technologies offering the same data rate.

Every year, apart from the number of subscribers joining the network, a certain number of the total connected subscribers leave it due to marketing, costs, and other requirements. This variation in the total number of connected subscribers is called user churn, $c$, and can change depending on the market and existing competitors. The user churn is defined as a Bernoulli process; the success probability decides if the churn occurs. Because the trials in each year are independent of each other, the overall process in the migration is memoryless and thus Markovian.

The level of churn is assumed to be $10 \%$ [18]. This user churn leads to lower revenue for the same operational expenditures because the services or the connection to the subscriber exists, but there is no monetary benefit from it. We do not consider the ITS MBSs as a part of churn because we assume that, once connected, the public ITS provider does not leave the network. With this assumption, the operator revenue in a single year, $t$, is defined as

$$
R_{t}\left(\gamma_{t}\right)=\sum_{q \in \mathrm{Sub}}(1-c)^{\gamma_{t}} n_{q, t} \cdot R_{q},
$$

where Sub $\in\{$ Residential, Business $\}$, and it takes values from Table I; $n_{q, t}$ is the number of subscribers of type $q$

TABLE I

YeARLY ARPU SUMmaRY, BASED ON [23]

\begin{tabular}{lccc}
\hline $\begin{array}{l}\text { Data Rate } \\
{[\mathrm{Mbps}]}\end{array}$ & $\begin{array}{c}\text { Residential } \\
\text { [C.U./year] }\end{array}$ & $\begin{array}{c}\text { Business } \\
\text { [C.U./year] }\end{array}$ & $\begin{array}{c}\text { ITS } \\
\text { [C.U./year] }\end{array}$ \\
\hline 20 & 3.6 & 3.6 & - \\
30 & 7.2 & 36 & - \\
50 & 10.8 & 84 & 84 \\
100 & 13.2 & 110 & 110 \\
\hline
\end{tabular}


who are connected to the network at year $t$ (calculated from the penetration curves shown in Fig. 3 ); $R_{q}$ is the per subscriber yearly revenue of type $q ; 0 \leq c \leq 1$ is the yearly churn rate of the subscribers; and $\gamma_{t}$ is a binary variable, which is defined as follows:

$$
\gamma_{t}= \begin{cases}1 & \text { if churn occurs } \\ 0 & \text { otherwise }\end{cases}
$$

\section{Expectimax-BAsed Migration Algorithm}

This section introduces the proposed AI-based migration algorithm. Here, we define the migration decision metric. Then, we introduce the modified Expectimax algorithm and apply it to a simple example.

Like the Expectimax algorithm, we also used three different nodes (maximizer, chance, and terminal), whose functions are briefly discussed in Section II.

\section{A. Proposed Decision Metric and Utility Function}

In this work, we choose the NPV [24] as our decisionmaking metric. NPV is an economic metric that shows the time value of money to evaluate long-term projects or project profitability, and it is defined as

$$
\mathrm{NPV}=\sum_{t=1}^{T_{\mathrm{NW}}} \mathrm{PV}_{t}
$$

where $t$ is the time iterator, $T_{\mathrm{NW}}$ is the network life cycle, and $\mathrm{PV}_{t}$ is the present value (PV) of the net cash flows at the time $t$. PV is defined as follows:

$$
\mathrm{PV}_{t}=\frac{R_{t}-I_{t}}{(1+d)^{t}}
$$

where $t$ is the time iterator indicating the current time period, $R_{t}$ is the revenue at time $t$ (cash inflow) as defined by Eq. (1), $d$ is the discount rate of the project, and $I_{t}$ is the investment made in time $t$ (cash outflow). This investment is the additional CAPEX required to migrate to a newer technology and the related OPEX costs for the deployed technology. We assume the discount rate to be fixed at $10 \%[13,20]$.

In the case where migrations occur in a given year, we need to model the CAPEX part of the investments. For this, we define an additional fixed cost inherent to migrating from technology $s$ to technology $s^{\prime}$ as

$$
\begin{aligned}
M_{s, s^{\prime}} & =\kappa_{i, j} \Delta_{\mathrm{CW}_{s, s^{\prime}}}+v_{p, q} \Delta_{\text {Equip }_{s, s^{\prime}}} \\
\forall s, \quad s^{\prime} & \in\{\text { Possible_Technologies }\}, \quad s \neq s^{\prime},
\end{aligned}
$$

where $\Delta_{\mathrm{CW}_{s, s^{\prime}}}$ is the difference in CAPEX civil works when there is a change of architecture, provided by the binary variable, $\kappa_{i, j}$, defined in Eq. (6), and $\Delta_{\text {Equip }_{s, s^{\prime}}}$ is the difference in CAPEX equipment when there is an upgrade of the delivered bandwidth, provided by the binary variable, $v_{p, q}$, defined in Eq. (7). These two CAPEX values, $\Delta_{\mathrm{CW}_{s, s^{\prime}}}$ and $\Delta_{\text {Equip }_{s, s^{\prime}}}$, follow the TCO cost modeling in [9]. These binary variables, $\kappa_{i, j}$ and $v_{p, q}$, distinguish which network upgrades have to be conducted: civil work, equipment, or both:

$$
\kappa_{i, j}=\left\{\begin{array}{l}
1 \\
\text { if } i \neq j \forall i, \quad j \in\{\mathrm{FTTCab}, \mathrm{FTTB}, \mathrm{FTTH}\} \\
0 \quad \text { otherwise }
\end{array},\right.
$$

$$
v_{p, q}= \begin{cases}1 & \text { if } p \neq q \forall p, q \in\{20,25,50,100\} \mathrm{Mbps} \\ 0 & \text { otherwise }\end{cases}
$$

Based on the definitions above, we define our Utility Function, $U$, for a current technology $s$ at year $t$ given a user churn, $\gamma_{t}$, for a terminal node (T.N.) and a maximizer node (M.N.), as follows:

$$
\begin{aligned}
& U\left(s, t, \gamma_{t}\right) \\
& = \begin{cases}\sum_{i=t}^{T_{\mathrm{NW}}} \frac{R_{i}\left(\gamma_{i}\right)-\mathrm{OPEX}_{i}}{(1+d)^{T_{\text {start }}}{ }^{i}} & \text { if T.N. } \\
\max _{s^{\prime}}\left[H\left(s^{\prime}, t+1\right)+\frac{R_{t}\left(\gamma_{t}\right)-\mathrm{OPEX}_{t}}{(1+d)^{T} \text { start }^{-t}}-\mu_{t} \cdot M_{s, s^{\prime}}\right] & \text { if M.N. }\end{cases}
\end{aligned}
$$

where $R_{i}\left(\gamma_{i}\right), \mathrm{OPEX}_{i}$, and $M_{s, s^{\prime}}$ are the revenue, yearly OPEX, and migration CAPEX, respectively. $\gamma_{i}$ is a binary variable ( $\gamma_{i}=1$ when user churn has occurred; $\gamma_{i}=0$ otherwise). $s$ is the current technology, and $s^{\prime}$ is the technology to be migrated to; $T_{\text {start }}$ is the starting year of the migration window; $d$ is the discount rate of the project; $\mu_{t}$ is a binary variable $\left(\mu_{t}=1\right.$ when migration takes place in year $t$; $\mu_{t}=0$ otherwise). The network life cycle is $T_{\mathrm{NW}}$, which can be any period of years longer than $T_{\text {mig. }} H\left(s^{\prime}, t+1\right)$ is the expected present value generated by the child maximizer nodes at depth $t+1$ for the next technology $s^{\prime}$.

At the maximizer nodes, the subtraction of $\mu_{t} \cdot M_{s, s^{\prime}}$ implicitly refers to the capital expenditures required for migration. The value at chance nodes is the weighted average of the value of its children, given by Eq. (9):

$$
H(s, t)=\sum_{\gamma_{t} \in\{0,1\}} \operatorname{Pr}\left(\gamma_{t}\right) \cdot U\left(s, t, \gamma_{t}\right)
$$

where $H(s, t)$ is the value of the chance node of state $s$ at depth $t$. Looking at Eqs. (8) and (9), it is evident that the algorithm is recursive in nature.

To model the uncertainty of churn, we define $\operatorname{Pr}\left(\gamma_{t}\right)$ as a two-state Markov chain with steady-state probabilities as defined in Eq. (10):

$$
\operatorname{Pr}\left(\gamma_{t}\right)=\left\{\begin{array}{ll}
0.9 & \text { if } \gamma_{t}=0 \\
0.1 & \text { if } \gamma_{t}=1
\end{array} .\right.
$$

\section{B. Proposed Search Tree Algorithm}

To explain the algorithm, let us use a simple example of a migration from ADSL (20 Mbps) involving two 
technologies (see Fig. 2): FTTCab_GPON_25 (referred to as PON1) and FTTB_XGPON_100 (referred to as PON2). The migration window, $T_{\mathrm{mig}}$, is set to 3 years, and the network life cycle, $T_{\mathrm{NW}}$, is set to 5 years. The final goal of the VIO is to find a technology that provides it with the maximum profitability across the entire $T_{\mathrm{NW}}$. Every year, for every possible migration, we have a maximizer node (blue rectangles with solid outlines in Fig. 4) and a chance node (red rectangle with dashed outline). For subsequent years, there can be either terminal nodes (green rectangles with dotted outline) or maximizer nodes again. We first discuss how the tree is built and then move to its evaluation using the utility function defined in Subsection IV.A.

1) Building the Search Tree: At the beginning of the migration period, a maximizer node is present and indicates the current state of migrations (no migrations). Each of its child nodes (chance nodes) is assigned a new technology to which migration is possible. For each chance node, there are two possibilities, namely, Churn and No_Churn, both having different utilities [refer to Eqs. (9) and (10)]. Figure 4 shows that PON2 Churn and No Churn in 2019 are terminal nodes instead of maximizer nodes. This happens because PON2 satisfies the goal of the VIO to provide $100 \mathrm{Mbps}$ to its subscribers.

Also, in 2019, PON1 has an option to migrate to either PON2 or not migrate at all. In the final year, all the technologies result in a terminal node because the tree reaches the end of the migration window. The flow of the tree building is a recursive approach and is implemented according to the pseudocode provided in Algorithm 1.

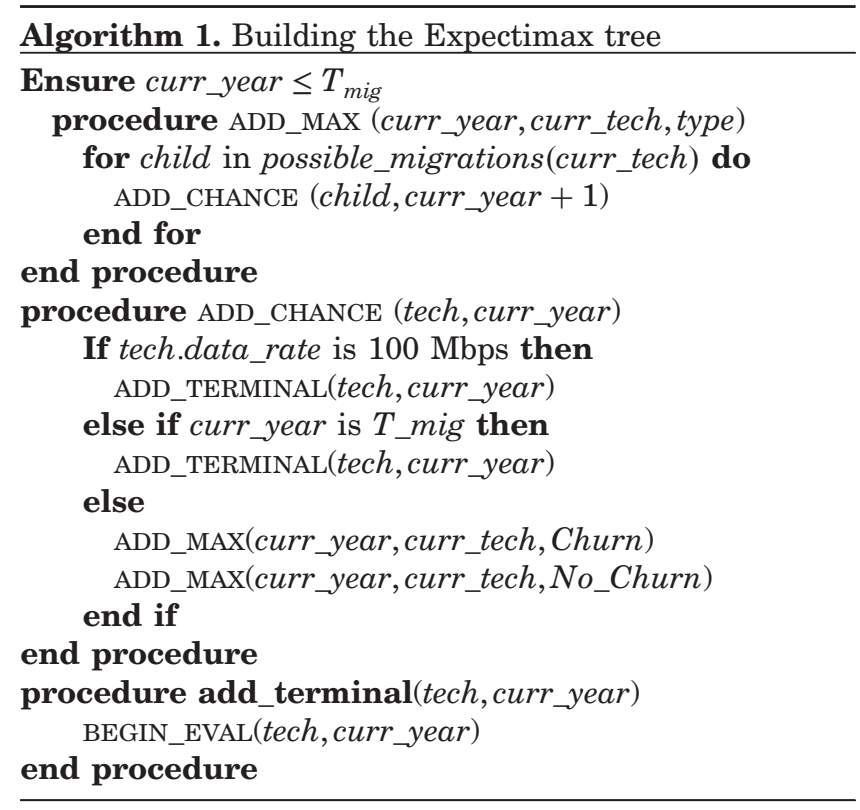

2) Evaluating the Search Tree: After the tree is built, the evaluation finds the migration path with the highest expected utility value. For explaining this evaluation, we refer to Fig. 5. The algorithm always starts from the terminal nodes and then traverses its way up. The values stored at the terminal nodes are the NPV values from that year to the end of $T_{\mathrm{NW}}$ [using Eq. (8)], assuming no further churn

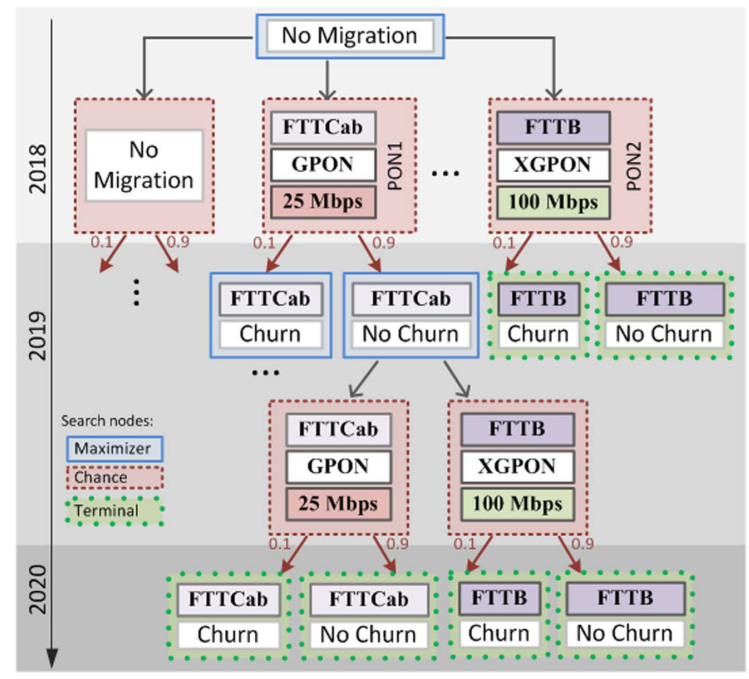

Fig. 4. Top-down building of search tree of depth 3 years and two technology choices. The tree is built recursively until a terminal condition is satisfied.

occurs, and the subscriber penetration follows the "realistic" curve. The chance nodes find the expected value of its children, which translates to the expected NPV from the current year to the end of the network life cycle [using Eq. (9)].

At every maximizer node, migration costs are subtracted from the accumulated NPV, which is seen from the PON1 maximizer node in year 2019. This is repeated until the top-most maximizer node is reached, where the node with the maximum accumulated NPV is chosen. This leads to the search tree choosing the migration path as PON1 in 2019 and PON2 in 2020 as the most profitable decision, with an accumulated NPV of 381 C.U. Algorithm 2 shows

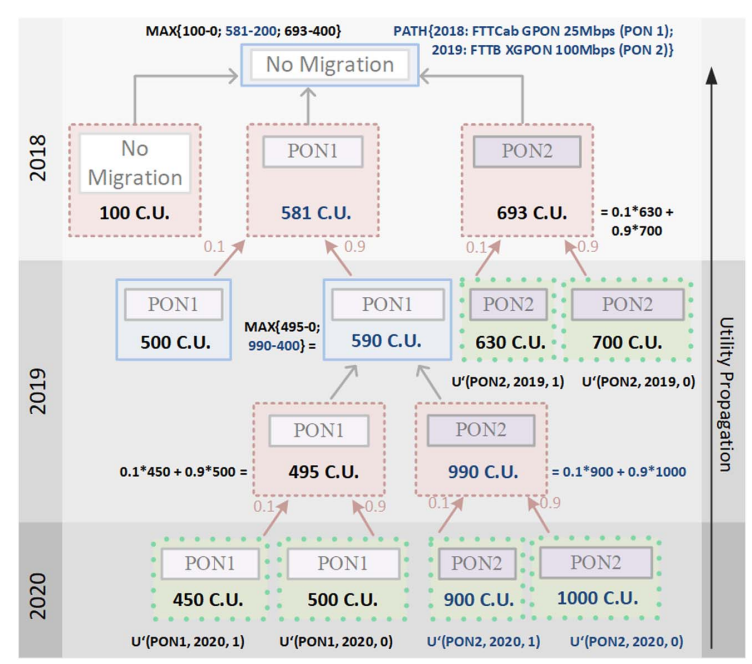

Fig. 5. Bottom-up evaluation of the utility function in the search tree reveals the migration path (in blue), which provides the highest NPV at the end of the network life cycle. 
how a built tree is evaluated, based on the current node being evaluated.

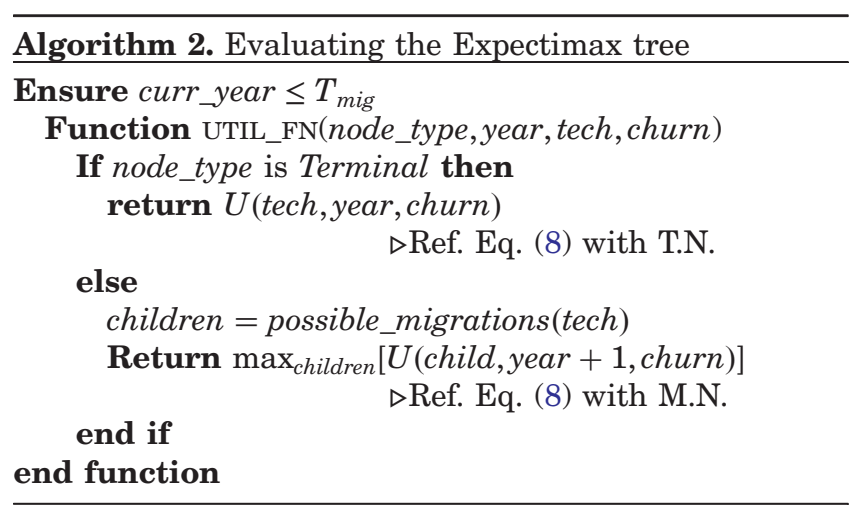

\section{Evaluation of the Proposed Migration Algorithm}

In our work, we have evaluated the performance of the proposed migration algorithm in a number of case studies. We looked into three types of deployment areas: dense urban (New York), urban (Munich), and suburban (Ottobrunn) [25].

Due to space limitations, we present the results of the urban deployment scenario in Munich (Germany). All the other results have been showcased and thoroughly discussed in Chapter 6 of [6]. An interested reader can also reproduce these results using the source code and input Excel files (including migration matrices) provided in [8].

As already mentioned, three demand types have been considered: residential, business, and public ITS base stations. The residential demands are defined by the building positions and the number of households per building, whereas the business demands are defined by the building positions and the density of the businesses in that area. For added security purposes, the business and ITS demands need to be sent on different wavelengths, which is supported by UDWDM and HPON technologies.

In the case of Munich, each building hosts a multiple dwelling unit (MDU), which connects an average of six to eight potential residential or business subscribers $[26,27]$. These subscribers have been divided into residential and business subscribers using the fixed percentage of business subscribers in a city [9,28]. In Munich, 7\% of the total buildings are considered to be business buildings [13]. The considered demands for the converged migration are 27,213 residential, 2049 business, and 2 ITS MBS.

All the presented results refer to the following scenario: area of $7 \mathrm{~km}^{2}$ in the center of Munich, migration starting in $T_{\text {start }}=2018$ and ending in 2027 (i.e., $T_{\text {mig }}=10$ years), $T_{\mathrm{NW}}$ is set to 20 years, churn rate of $c=10 \%$, and churn probability $\operatorname{Pr}\left(\gamma_{t}\right)=0.1$. The technological scenario in our case was migrated from the existing copper, i.e., ADSL2+, network to a future-proof PON architecture $[1,18]$.

The migration goal for the residential and business subscribers is to offer at least $100 \mathrm{Mbps}$ per household or business by 2025. This requirement was dictated by the EU
Broadband Regulation Policy on Digital Single Market [19]. The ITS base stations, although not governed by any EU regulatory policy, have a technical requirement of at least a $50 \mathrm{Mbps}$ data rate, to support current public ITS demands [21]. However, a higher data rate of $100 \mathrm{Mbps}$ was assumed to be favorable in the case of a future increase in public ITS traffic.

\section{A. Pure Residential Migration}

Let us first consider a purely residential scenario, where all the demands in the network are residential subscribers, i.e., households, whose tariffs are provided in Table I.

Out of the 13 different PON technologies that can be derived from the migration tree shown in Fig. 2, Fig. 6 shows the per subscriber CAPEX in C.U. of selected technologies, all of which offer at least $100 \mathrm{Mbps}$ to the subscriber. The CAPEX costs are divided into components like civil works, fiber laying costs, and equipment costs at various locations like the central office (CO), remote nodes (RNs), and buildings. We observed that FTTH technologies are more expensive than non-FTTH ones, with FTTH_XGPON_100 being the most expensive technology to deploy (11.18 C.U. per subscriber). Due to a single-stage deployment and a high subscriber density in urban areas, UDWDM-based FTTB/FTTH technologies have lower civil works cost as compared with XGPON-based FTTB/FTTH technologies.

From Fig. 7, we see that FTTH technologies have the least OPEX, about 0.6 C.U. per subscriber. This is because the subscribers pay for the rent and energy costs of the ONU. The highest OPEX of about 1 C.U. per subscriber per year is from FTTB_UDWDM_100 because the energy and rent costs of expensive UDWDM ONUs are borne by the VIO. To supplement the migration algorithm, we fix the per subscriber OPEX in ADSL (copper) to 0.25 C.U. The OPEX calculations in our work are based on the model and data provided in [16]. The results of the expected NPV and the migration path for each of the different subscriber penetration curves are shown in Table II.

From the results in Table II, we observe that due to low ARPU for residential subscribers, the VIO cannot have a higher NPV in a purely residential deployment, unless

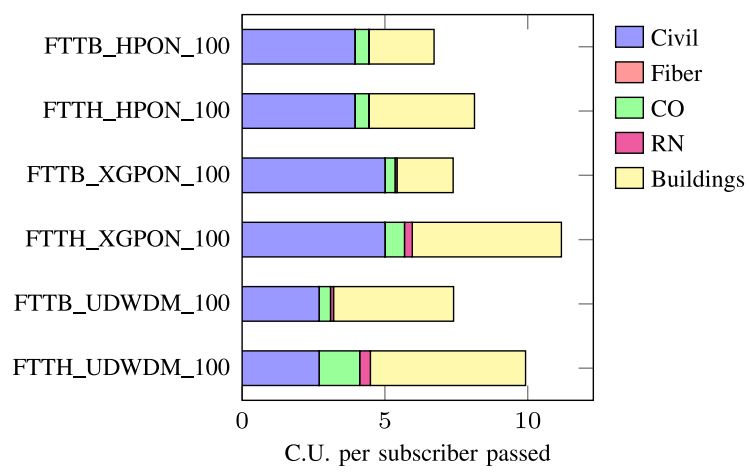

Fig. 6. CAPEX categories of selected deployments in the Munich pure residential scenario offering $100 \mathrm{Mbps}$ to subscribers. 


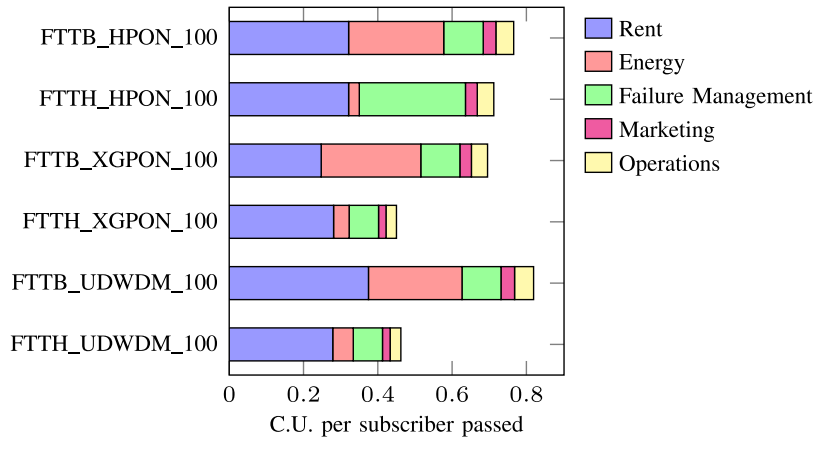

Fig. 7. OPEX categories of selected deployments in the Munich pure residential scenario offering $100 \mathrm{Mbps}$ to subscribers in year 2018 .

the subscriber penetration is aggressive. We see that when only FTTH technologies provide $100 \mathrm{Mbps}$, the NPV is 20\%-50\% lower because non-FTTH technologies with 100 Mbps (like FTTB_UDWDM_100) have a higher return on investment. We also observe that the algorithm suggests migrations in the beginning of the migration window, to get more revenue from the subscribers. This behavior is also recorded by the heuristic optimization models of [11].

Overall, in the worst case of a conservative subscriber penetration rate, coupled with only FTTH technologies providing $100 \mathrm{Mbps}$ data rates, early migrations to a hybrid PON optical architecture result in at least a minimally positive NPV.

\section{B. Converged Migration}

Let us now consider a converged scenario of all different demands: residential, business, and public ITS MBSs have to be satisfied. First, the planning and cost evaluation are performed, and the results show that both CAPEX and OPEX for every architecture in the converged scenario are between $5 \%$ and $10 \%$ higher than in the pure residential scenario. While deploying the network, it is mandatory that the business subscribers and residential subscribers do not share equipment for security reasons [23].
To visualize better a converged network deployment, let us focus on the example of FTTH_HPON_100, which has been depicted in Fig. 8. To meet the demands, every 10 Gbps card at the OLT is connected to a single-arrayed waveguide grating (AWG) at the first RN. This device splits the optical signal from the feeder fiber to up to 80 different wavelengths that can be either split further into $100 \mathrm{Mbps}$ connections at the second RNs or can be connected directly to ITS LTE MBSs.

In this deployment, additional infrastructure and equipment were added to ensure that each business building (consisting of six to eight business subscribers) gets its own wavelength, in accordance with the service level agreements (SLAs) that justify the higher price [23]. Due to these factors, the NPV is on average 1.6-7.5 times higher for the converged case in all different scenarios, as compared with a pure residential scenario. The results from the migration algorithm are shown in Table III.

In cases where migrations to FTTx technologies are allowed, FTTB_HPON_100 is preferred due to its highest NPV. Compared with the pure residential scenario, the algorithm selects HPON for "conservative" penetration curves because they are more cost effective as compared with GPON and UDWDM technologies. Among FTTH technologies, migration to FTTH_UDWDM_100 is preferred

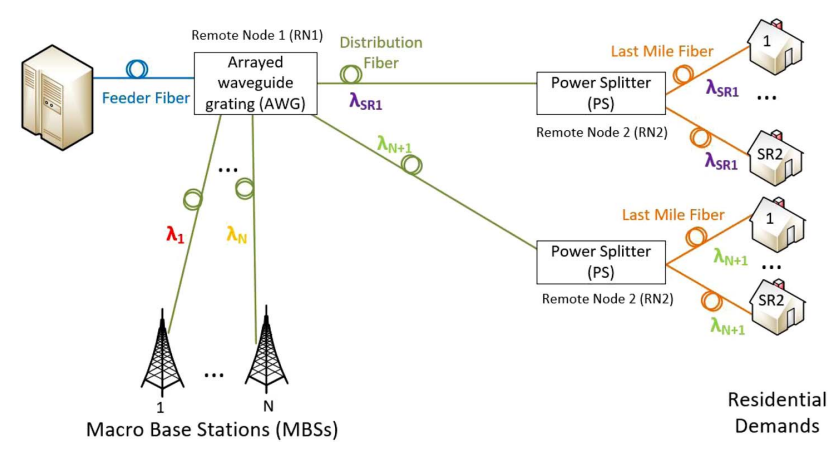

Fig. 8. Example deployment of a two-stage FTTH_HPON_100 with a 1:80 AWG at RN1 and 1:16 power splitters at RN2 in a converged scenario.

TABLE II

Technologies Deployed in Each Scenario for the Munich Pure Residential Scenario

\begin{tabular}{lcc}
\hline FTTCab/FTTB/FTTH Provide $100 \mathrm{Mbps}$ & & \\
\hline Penetration Curve & FTTx Migration Path & Net Present Value [C.U.] \\
\hline Conservative & 2019: FTTB_UDWDM_50 & $\mathbf{9 3 , 8 3 7}$ \\
Realistic & 2020: FTTB_UDWDM_100 & $\mathbf{1 8 0 , 1 6 1}$ \\
Aggressive & 2019: FTTB_UDWDM_50 & $\mathbf{8 0 2 0}$ FTTB_UDWDM_100 \\
\hline Only FTTH Provides $100 \mathrm{Mbps}$ & 2019: FTTB_UDWDM_100 & $\mathbf{8 8 6 , 7 8}$ \\
\hline Penetration Curve & & Net Present Value [C.U.] \\
Conservative & FTTH Migration Path & $\mathbf{1 3 , 3 1 3}$ \\
Realistic & 2019: FTTH_HPON_100 \\
Aggressive & 2019: FTTH_HPON_100 & $\mathbf{1 0 0 , 6 7 9}$ \\
\hline
\end{tabular}


TABLE III

Technologies Deployed in Each Migration Scenario for the Munich Converged Scenario FTTCab/FTTB/FTTH Provide 100 Mbps

\begin{tabular}{lcc}
\hline Penetration Curve & FTTx Migration Path & Net Present Value [C.U.] \\
\hline Conservative & $2019:$ FTTB_HPON_50 & $\mathbf{1 9 0 , 0 6 9}$ \\
Realistic & $2020:$ FTTB_HPON_100 & $\mathbf{3 2 5 , 1 3 2}$ \\
Aggressive & $2019:$ FTTB_HPON_50 & $\mathbf{1 , 4 2 9 , 2 7 9}$ \\
\hline Only FTTH Provides $100 \mathrm{Mbps}$ & 2019: FTTB_UDWDM_100 & Net Present Value [C.U.] \\
\hline Penetration Curve & & $\mathbf{9 9 , 8 8 5}$ \\
\hline Conservative & FTTH Migration Path & $\mathbf{2 3 6 , 4 5 2}$ \\
Realistic & 2019: FTTH_UDWDM_100 \\
Aggressive & 2019: FTTH_UDWDM_100 & $\mathbf{1 , 3 5 2 , 0 0 4}$ \\
\hline
\end{tabular}

because the high operational costs are offset by charging a higher tariff from business and ITS subscribers. FTTH_ HPON_100, which was the preferred technology in the residential scenario, loses out marginally to FTTH UDWDM_100 in this case, due to higher ONU costs in the hybrid PON technology.

\section{Sensitivity Analysis}

The sensitivity analysis has been applied to the converged planning scenario of the Munich (Urban) area. The other scenarios have also been implemented, tested, and found to behave in the same way as the one presented in this section. Here, we chose a positive scenario, where there are no constraints on when or to which technology to migrate. Because different studies have different units of currency, like pounds, euros, and cost units, we converted all the currencies into cost units using the current currency conversion. Here 1 C.U. is fixed at 50 euros and 44.97 GBP.

1) Component Costs: Because technoeconomic studies are specific to the costs involved in an area, different cost models have different expected NPVs. In this study, we took the costs mentioned in three different studies, namely, Rokkas [28] (deploying GPON-based technology in a generic urban area), Phillipson [29] (deploying HPONbased technology in a Dutch city), and BSG [30] (FTTCabbased deployment in London). As a further contribution, we collected data from each of these different studies and undertook the cost modeling for all the different deployment technologies. We then compared all the technologies with each other and also with the base case, which are the values taken from [16].

For each of the four studies, we ran the migration algorithm and found the expected NPV and the migration steps. Both [28] and [29] are comparable in terms of cost to the OASE base model [18]. Hence, the results can vary according to the costs. Table IV gives a list of major component costs from each of the studies. Because all components used in our work were not mentioned in every study, we assumed these components to follow model trends. Figure 9 shows the per subscriber CAPEX in cost units for selected $100 \mathrm{Mbps}$ deployments in a converged scenario. Three of the four studies have comparable costs, with the only exception being BSG.

We see that as the component costs increase, the NPV decreases, which is the expected behavior. As seen in Table V, except for the OASE base case, the other

TABLE IV

Per Unit Component Costs From OASE [18], Phillipson [29] Rokkas [28], and BSG [30]

\begin{tabular}{|c|c|c|c|c|}
\hline Component & OASE [C.U.] & Phillipson [C.U.] & Rokkas [C.U.] & BSG [C.U.] \\
\hline Fiber duct & $1.12 / \mathrm{m}$ & $0.54 / \mathrm{m}$ & $0.7 / \mathrm{m}$ & $1.42 / \mathrm{m}$ \\
\hline Fiber & $0.02 / \mathrm{m}$ & $0.006 / \mathrm{m}$ & $0.006 / \mathrm{m}$ & $0.192 / \mathrm{m}$ \\
\hline GPON OLT card & 40 & 50 & 70 & 288 \\
\hline XGPON OLT card & 80 & $55^{a}$ & 200 & $300^{a}$ \\
\hline WDM OLT port card & 8.8 & $60^{a}$ & 200 & $350^{a}$ \\
\hline Power splitter & 1.8 & $2^{a}$ & 10 & $1.4^{a}$ \\
\hline AWG & 2.2 & $2^{a}$ & $12^{a}$ & $2^{a}$ \\
\hline DSLAM+Cabinet & 124 & 220 & 300 & 294 \\
\hline GPON ONU & 1 & 5 & 2 & 1.6 \\
\hline XGPON ONU & 1.8 & $5^{a}$ & 4 & $1.8^{a}$ \\
\hline WDMPON ONU & 2.3 & $5^{a}$ & $5^{a}$ & $2.3^{a}$ \\
\hline HPON ONU & 3.1 & $5.5^{a}$ & 5 & $3.1^{a}$ \\
\hline
\end{tabular}

${ }^{a}$ Assumed according to model trends. 


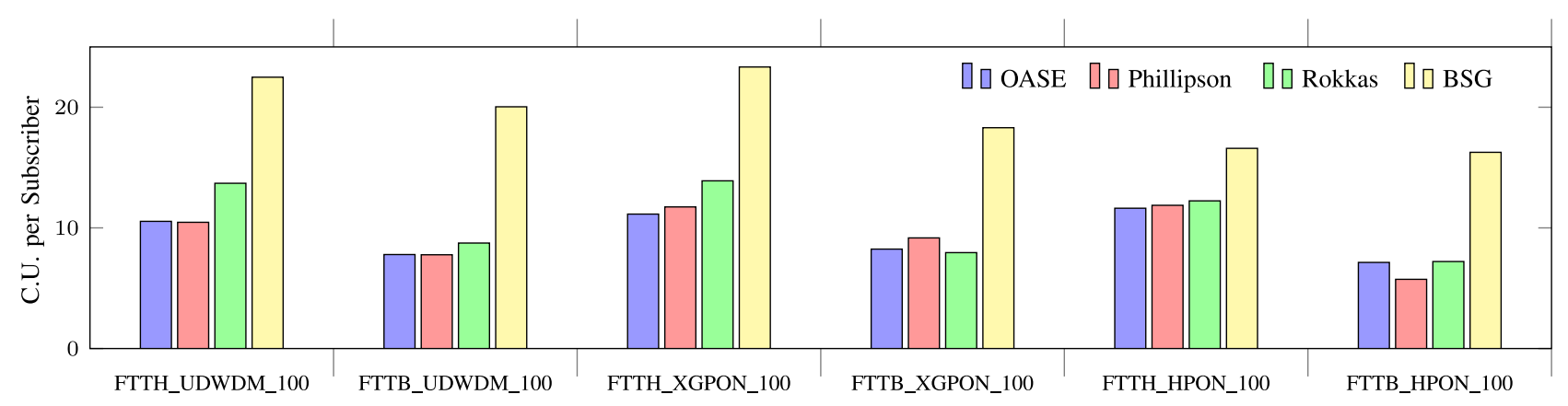

Fig. 9. CAPEX of selected deployments for different component costs in a converged scenario (OASE [18], Phillipson [29], Rokkas [28], BSG [30]).

TABLE V

Migration Results of Different Component Costs

\section{OASE [18]}

\begin{tabular}{|c|c|c|}
\hline Penetration Curve & FTTx Migration Path & Net Present Value [C.U.] \\
\hline Conservative & $\begin{array}{l}\text { 2019: FTTB_HPON_50 } \\
\text { 2020: FTTB_HPON_100 }\end{array}$ & 190,069 \\
\hline Realistic & $\begin{array}{l}\text { 2019: FTTB_HPON_50 } \\
\text { 2020: FTTB_HPON_100 }\end{array}$ & 325,132 \\
\hline Aggressive & 2019: FTTB_UDWDM__100 & $1,429,279$ \\
\hline \multicolumn{3}{|l|}{ Phillipson [29] } \\
\hline Penetration Curve & FTTx Migration Path & Net Present Value [C.U.] \\
\hline $\begin{array}{l}\text { Conservative } \\
\text { Realistic } \\
\text { Aggressive }\end{array}$ & $\begin{array}{l}\text { 2019:FTTB_HPON_100 } \\
\text { 2019:FTTB_HPON_100 } \\
\text { 2019:FTTB_HPON_100 }\end{array}$ & $\begin{array}{c}122,363 \\
221,742 \\
1,055,627\end{array}$ \\
\hline \multicolumn{3}{|l|}{ Rokkas [28] } \\
\hline Penetration Curve & FTTx Migration Path & Net Present Value [C.U.] \\
\hline $\begin{array}{l}\text { Conservative } \\
\text { Realistic } \\
\text { Aggressive }\end{array}$ & $\begin{array}{l}\text { 2019:FTTB_HPON_100 } \\
\text { 2019:FTTB_HPON_100 } \\
\text { 2019:FTTB_HPON_100 }\end{array}$ & $\begin{array}{c}75,805 \\
175,184 \\
1,009,069\end{array}$ \\
\hline \multicolumn{3}{|l|}{ BSG [30] } \\
\hline Penetration Curve & FTTx Migration Path & Net Present Value [C.U.] \\
\hline $\begin{array}{l}\text { Conservative } \\
\text { Realistic } \\
\text { Aggressive }\end{array}$ & $\begin{array}{c}\text { No Migrations } \\
\text { No Migrations } \\
\text { 2019: FTTB_HPON_100 }\end{array}$ & $\begin{array}{c}37,421 \\
53,510 \\
769,030\end{array}$ \\
\hline
\end{tabular}

components choose FTTB_HPON_100 as the final technology and prefer early migrations to reap maximum benefits. The only exception to this is the BSG costs in conservative and realistic scenarios, which suggests not undertaking any migrations. This is because of the high costs involved and no change in the ARPU. Because the BSG study was done for an expensive and densely populated city like London, it is possible that the ARPU would be higher than what is used in our analysis.

2) OPEX: The final part of the sensitivity analysis includes an OPEX study. In many technoeconomic works including [9] and [13], OPEX is considered as a fractional quantity of CAPEX, which was originally modeled in [31]. This is because technoeconomic researchers do not have access to component-specific data like mean time to repair, energy consumption, component footprint, and technician salaries.

Here, we choose two different OPEX models. The base model is already defined in Section III. The percentagebased OPEX model is taken from [13], where the OPEX of a technology $t$ is defined as follows:

$$
\mathrm{OPEX}_{t}=0.1 * C_{\mathrm{Elec}_{t}}+0.01 * C_{\mathrm{CW}_{t}}
$$

where $C_{\mathrm{Elec}_{t}}$ and $C_{\mathrm{CW}_{t}}$ are the CAPEX of the electronic and civil works of a technology $t$, respectively.

As seen in Eq. (11), the OPEX is directly proportional to the CAPEX values shown in Fig. 6. For most technologies, 
TABLE VI

Migration Results for Different OPEX Models

\begin{tabular}{lcr}
\hline Base OPEX Model & & \\
\hline Penetration Curve & FTTx Migration Path & Net Present Value [C.U.] \\
\hline Conservative & $2019:$ FTTB_HPON_50 & $\mathbf{1 5 2 , 4 0 1 . 5 1}$ \\
Realistic & $2020:$ FTTB_HPON_100 & $\mathbf{2 7 2 , 7 2 6 . 5 5}$ \\
Aggressive & $2019:$ FTTB_HPON_50 & $\mathbf{1 , 2 8 2 , 6 3 1 . 5 4}$ \\
\hline Percentage OPEX Model & 2020: FTTB_HPON_100 & 2019: FTTH_UDWDM_100 \\
\hline Penetration Curve & & Net Present Value [C.U.] \\
\hline Conservative & FTTx Migration Path & $\mathbf{2 1 6 , 0 0 7 . 4 7}$ \\
Realistic & $2019:$ FTTB_HPON_50 & \\
Aggressive & $2020:$ FTTB_HPON_100 & $\mathbf{3 5 9 , 3 7 2 . 6 5}$ \\
\hline
\end{tabular}

the percentage-based OPEX is cheaper per subscriber connected. However, in the case of FTTH-based technologies, the newer OPEX model is between 0.25 and 1 C.U. higher for every subscriber connected. Hence, the benefits of OPEX savings due to lower energy costs in FTTH architectures are not considered in this fraction-based model.

From Table VI, we infer that for a rough analysis, a percentage-based OPEX model could be considered, keeping in mind the risk of underestimating the cost factors of various technologies.

\section{Conclusions and OuTloOK}

Network operators have to be timely in upgrading their access network infrastructure to provide the required services and stay profitable. This motivation defines our approach to the complex strategic multiperiod migration problem. We put the primary goal of the operator, profitability in terms of maximizing the NPV, as our utility function for the migration algorithm. For the adequate calculation of the utility, we calculate the realistic deployment and operations costs and take into account subscriber penetration, subscriber churn, and realistic revenue.

In this paper, we have proposed a migration algorithm based on a modified Expectimax search to find the migration paths. We show that the proposed flexible final state results in up to $50 \%$ profitability increase over typical state-of-the-art fixed-state migrations. We also avoid overestimating the revenue by including the user churn in our model. Finally, we have validated our assumptions with the sensitivity analysis.

The proposed migration algorithm is flexible and can be used for a migration study with any user-defined migration window. It could be improved with a heuristic-based pruning of the search tree to reduce time complexity. For a future upgrade, the churn probability can be modeled using a more realistic model. Real-world access network migration data may be used to get more accurate estimates or to develop a generalized heuristic model. Furthermore, percentage-based mixed cases could be added to the model as an input, and the results of these partial migration scenarios could be evaluated. The revenue model can also be further investigated for optimal results. Finally, reliable predictions can be made using machine-learning algorithms, like random forest, if sufficient migration data are present.

\section{ACKNOWLEDGMENT}

This work has received funding from the German Research Foundation (DFG) under grant numbers MA6529/2-1 and KE1863/4-1.

\section{REFERENCES}

[1] FTTH Council Europe, "Case studies collection," Feb. 2015.

[2] K. McMahon and P. Salant, "Strategic planning for telecommunications in rural communities," Rural Dev. Perspect., vol. 14, pp. 2-7, 1999.

[3] R. Romero Reyes, R. Zhao, and C. Mas Machuca, "Advanced dynamic migration planning toward FTTH," IEEE Commun. Mag., vol. 52, no. 1, pp. 77-83, 2014.

[4] D. Klein, "Lecture notes in CS 188: Artificial intelligence Fall 2010 Expectimax search,” Berkeley, California, 2010.

[5] S. J. Russell and P. Norvig, Artificial Intelligence: A Modern Approach, 3rd ed., Pearson Education, Inc., 2010.

[6] S. K. Patri, E. Grigoreva, and C. Mas Machuca, "Converged network migration planning," Tech. Rep., 2018 [Online]. Available: https://mediatum.ub.tum.de/doc/1459271/1459271.pdf.

[7] E. Grigoreva, "Automated geography-based fixed network planning tool," 2018 [Online]. Available: https://github.com/ EGrigoreva/FixedNetworkPlanningTool.

[8] S. K. Patri, "ExpectiMigPlan: An AI-based network planning tool," 2018 [Online]. Available: https://github.com/SaiPatri/ ExpectiMigPlan.

[9] S. van der Merwe, C. G. Gruber, Y. Grigoreva, and T. Kessler, "A model-based techno-economic comparison of optical access technologies," in GLOBECOM Workshops, 2009, pp. 1-6.

[10] A. Mitcsenkov, M. Kantor, K. Casier, B. Lannoo, K. Wajda, J. Chen, and L. Wosinska, "Geometric versus geographic models for the estimation of an FTTH deployment," Telecommun. Syst., vol. 54, no. 2, pp. 113-127, 2013. 
[11] S. Türk, X. Liu, R. Radeke, and R. Lehnert, "Particle swarm optimization of network migration planning," in IEEE Global Telecommunications Conf. (GLOBECOM), 2013, pp. 2230-2235.

[12] S. Türk, J. Noack, R. Radeke, and R. Lehnert, "Strategic migration optimization of urban access networks using metaheuristics," in 26th Int. Teletraffic Congr. (ITC), 2014.

[13] M. Tahon, S. Verbrugge, D. Colle, M. Pickavet, P. J. Willis, and P. Botham, "Migration to next generation access networks: A real option approach," in 16th Annu. Int. Conf. on Real Options, 2012, pp. 1-11.

[14] J. Mun, "Real options analysis versus traditional DCF valuation in layman's terms," in Managing Enterprise Risk: What the Electric Industry Experience Implies for Contemporary Business, 2006, pp. 75-106.

[15] M. Forzati, C. Mattsson, K. Wang, and C. P. Larsen, "The uncaptured value of FTTH networks," in 13th Int. Conf. on Transparent Optical Networks, June 2011, pp. 1-4.

[16] OASE, "Technical assessment and comparison of nextgeneration optical access system concepts/OASE," OASE Deliverable 4.2.1, 2011, pp. 40-68.

[17] M. Van der Wee, S. Verbrugge, M. Tahon, D. Colle, and M. Pickavet, "Evaluation of the techno-economic viability of point-to-point dark fiber access infrastructure in Europe," J. Opt. Commun. Netw., vol. 6, no. 3, p. 238-249, 2014.

[18] OASE, "OASE value network evaluation," OASE Deliverable $6.3,2013$.

[19] European Commission on Digital Single Market, "Strategy \& policy on broadband Europe: Digital single market," 2014.

[20] K. Casier, "Techno-economic evaluation of a next generation access network deployment in a competitive setting," Ph.D. dissertation, Ghent University, 2009.

[21] E. Grigoreva, C. Mas Machuca, and W. Kellerer, "Optical backhaul network planning for DSRC-based public intelligent transportation system: A case study," July 2016, pp. $1-4$.
[22] Environmental Systems Research Institute, “ArcGISDesktop, Release 10.3.1," 2011 [Online]. Available: http://desktop.arcgis.com/.

[23] FTTH Council, "FTTH business guide," Fiber to the Home Council Europe Tech. Rep., 2016.

[24] D. S. Remer and A. P. Nieto, "A compendium and comparison of 25 project evaluation techniques. Part 1: Net present value and rate of return methods," Int. J. Prod. Econ., vol. 42, no. 1, pp. 79-96, 1995.

[25] A. Mitcsenkov, M. Kantor, K. Casier, B. Lannoo, K. Wajda, J. Chen, and L. Wosinska, "Geographic model for cost estimation of FTTH deployment: Overcoming inaccuracy in uneven-populated areas," in Asia Communications and Photonics Conf. and Exhibition (ACP), 2010, pp. 397-398.

[26] JLL, "Residential city profile Munich," Tech. Rep., 2016, p. 1.

[27] "New York City mandatory inclusionary housing," Tech. Rep., Government of New York City, 2018.

[28] T. Rokkas, "Techno-economic analysis of PON architectures for FTTH deployments: Comparison between GPON, XGPON and NG-PON2 for a Greenfield operator," in Conf. of Telecommunication, Media and Internet Techno-Economics (CTTE), 2015, pp. 1-8.

[29] F. Phillipson, C. Smit-Rietveld, and P. Verhagen, "Fourth generation broadband delivered by hybrid FTTH solutionA techno-economic study," J. Opt. Commun. Netw., vol. 5, no. 11, pp. 1328-1342, 2013.

[30] A. Mason, "The costs of deploying fibre-based next-generation broadband infrastructure," Final Rep., Broadband Stakeholder Group, Sept. 2008, Ref: 12726-371.

[31] S. Verbrugge, D. Colle, M. Pickavet, P. Demeester, S. Pasqualini, A. Iselt, A. Kirstädter, R. Hülsermann, F.-J. Westphal, and M. Jäger, "Methodology and input availability parameters for calculating OpEx and CapEx costs for realistic network scenarios," J. Opt. Netw., vol. 5, no. 6, pp. 509-520, 2006. 